\title{
Utterance Intent Classification of a Spoken Dialogue System with Efficiently Untied Recursive Autoencoders
}

\author{
Tsuneo Kato \\ Doshisha University \\ Ryosuke Sumitomo \\ KDDI Research, Inc.
}

\author{
Atsushi Nagai \\ Doshisha University \\ Jianming Wu \\ KDDI Research, Inc.
}

\author{
Naoki Noda \\ Doshisha University \\ Seiichi Yamamoto \\ Doshisha University
}

\begin{abstract}
Recursive autoencoders (RAEs) for compositionality of a vector space model were applied to utterance intent classification of a smartphone-based Japanese-language spoken dialogue system. Though the RAEs express a nonlinear operation on the vectors of child nodes, the operation is considered to be different intrinsically depending on types of child nodes. To relax the difference, a data-driven untying of autoencoders (AEs) is proposed. The experimental result of the utterance intent classification showed an improved accuracy with the proposed method compared with the basic tied RAE and untied RAE based on a manual rule.
\end{abstract}

\section{Introduction}

A spoken dialogue system needs to estimate the utterance intent correctly despite of various oral expressions. It has been a basic approach to classify the result of automatic speech recognition (ASR) of an utterance into one of multiple predefined intent classes, followed with slot filling specific to the estimated intent class.

There have been active studies on word embedding techniques (Mikolov et al., 2013), (Pennington et al., 2014), where a continuous real vector of a relatively low dimension is estimated for every word from a distribution of word co-occurence in a large-scale corpus, and on compositionality techniques (Mitchell and Lapata, 2010), (Guevara，2010), which estimate real vectors of phrases and clauses through arithmetic operations on the word embeddings. Among them, a series of compositionality models by Socher, such as recursive autoencoders (Socher et al., 2011), matrix-vector model which models the dependencies explicitly (Socher et al., 2012), compositional vector grammar which combines a probabilistic context free grammar (PCFG) parser with compositional vectors (Socher et al., 2013a) and the neural tensor network (Socher et al., 2013b) are gaining attention. The methods which showed effectiveness in polarity estimation, sentiment distribution and paraphrase detection are effective in utterance intent classification task (Guo et al., 2014), (Ravuri and Stolcke, 2015). The accuracy of intent classification should improve if the compositional vector gives richer relations between words and phrases compared to thesaurus combined with a conventional bag-of-words model.

Japanese, an agglutative language, has a relatively flexible word order though it does have an underlying subject-object-verb (SOV) order. In colloquial expressions, the word order becomes more flexible. In this paper, we applied the recursive autoencoder (RAE) to the utterance intent classification of a smartphone-based Japaneselanguage spoken dialogue system. The original RAE uses a single tied autoencoder (AE) for all nodes in a tree. We applied multiple AEs that were untied depending on node types, because the operations must intrinsically differ depending on the node types of word and phrases. In terms of syntactic untying, the convolutional vector grammar (Socher et al., 2013a) introduced syntactic untying. However, a syntactic parser is not easy to apply to colloquial Japanese expressions.

Hence, to obtain an efficient untying of AEs, we propose a data-driven untying of AEs based on a regression tree. The regression tree is formed to reduce the total error of reconstructing child nodes with AEs. We compare the accuracies of utterance intent classification among the RAEs of a single tied AE, AEs untied with a manually defined rule, and AEs untied with a data-driven split method. 
Table 1: Relative frequency distribution of utterance intent classes

\begin{tabular}{lrl}
\hline intent class tag & freq & sample utterance (translation) \\
\hline CheckWeather & 20.4 & How's the weather in Tokyo now? \\
Greetings & 16.5 & Good morning. \\
AskTime & 11.3 & What time is it now? \\
CheckSchedule & 7.2 & Check today's schedule. \\
SetAlarm & 5.7 & Wake me up at 6AM tomorrow. \\
Thanks & 3.6 & Thank you. \\
Yes & 3.1 & Yes. \\
Goodbye & 2.4 & Good night. \\
WebSearch & 2.2 & Search (keyword) \\
Praise & 2.2 & You are so cute. \\
Time & 1.9 & Tomorrow. \\
MakeFun & 1.6 & Stupid. \\
GoodFeeling & 0.9 & I'm fine. \\
BadFeeling & 0.8 & I am tired \\
CheckTemp & 0.8 & What is the temperature today? \\
BackChannel & 0.7 & Sure. \\
AddSchedule & 0.7 & Schedule a party at 7 on Friday. \\
FortuneTeller & 0.7 & Tell my fortune today. \\
Call & 0.6 & Ho. \\
No & 0.6 & No way. \\
\hline \multicolumn{2}{c}{ freq. : relative frequency distribution in percent. } \\
\end{tabular}

\section{Spoken Dialog System on Smartphone}

The target system is a smartphone-based Japaneselanguage spoken dialog application designed to encourage users to constantly use its speech interface. The application adopts gamification to promote the use of interface. Variations of responses from an animated character are largely limited in the beginning, but variations and functionality are gradually released along with the use of the application. Major functions include weather forecast, schedule management, alarm setting, web search and chatting.

Most of user utterances are short phrases and words, with a few sentences of complex contents and nuances. The authors reviewed ASR log data of 139,000 utterances, redifined utterance intent classes, and assigned a class tag to every utterance of a part of the data. Specifically, three of the authors annotated the most frequent 3,000 variations of the ASR log, which correspond to 97,000 utterances i.e. $70.0 \%$ of the total, redefined 169 utterance intent classes including an others class, and assigned a class tag to each 3,000 variations.

Frequent utterance intent classes and their relative frequency distribution are listed in Table 1. A small number of major classes occupy more than half of the total number of utterances, while there are a large number of minor classes having small portions.

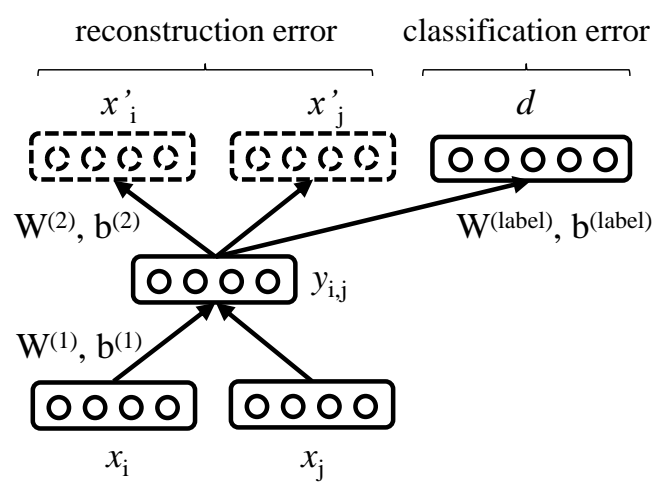

Figure 1: Model parameters and error functions of the recursive autoencoder

\section{Intent Class Estimation based on Untied RAE}

\subsection{Training of Basic RAE}

Classification based on RAE takes word embeddings as leaves of a tree and applies an $\mathrm{AE}$ to neighboring node pairs in a bottom-up manner repeatedly to form a tree. The RAE obtains vectors of phrases and clauses at intermediate nodes, and that of a whole utterance at the top node of the tree. The classification is performed by another softmax layer which takes the vectors of the words, phrases, clauses and whole utterance as inputs and then outputs an estimation of classes.

An AE applies a neural network of model parameters: weighting matrix $W^{(1)}$, bias $b^{(1)}$ and activation function $f$ to a vector pair of neighboring nodes $x_{i}$ and $x_{j}$ as child nodes, and obtains a composition vector $y_{(i, j)}$ of the same dimension as a parent node.

$$
y_{(i, j)}=f\left(W^{(1)}\left[x_{i} ; x_{j}\right]+b^{(1)}\right)
$$

The AE applies another neural network of an inversion which reproduces $x_{i}$ and $x_{j}$ as $x_{i}^{\prime}$ and $x_{j}^{\prime}$ from $y_{(i, j)}$ as accurately as possible. The inversion is expressed as equation (2).

$$
\left[x_{i}^{\prime} ; x_{j}^{\prime}\right]=f\left(W^{(2)} y_{(i, j)}+b^{(2)}\right)
$$

The error function is reconstruction error $E_{r e c}$ in (3).

$$
E_{\text {rec }}=\frac{1}{2}\left|\left[x_{i}^{\prime} ; x_{j}^{\prime}\right]-\left[x_{i} ; x_{j}\right]\right|^{2}
$$

The tree is formed in accordance with a syntactic parse tree conceptually, but it is formed by greedy search minimizing the reconstruction error in reality. Among all pairs of neighboring nodes 
at a time, a pair that produces the minimal reconstruction error $E_{r e c}$ is selected to form a parent node.

Here, the AE applied to every node is a single common one, specifically, a set of model parameters $W^{(1)}, b^{(1)}, W^{(2)}$ and $b^{(2)}$. The set of model parameters of the tied RAE is trained to minimize the total of $E_{r e c}$ for all the training data.

The softmax layer for intent classification takes the vectors of nodes as inputs, and outputs posterior probabilities of $K$ units. It outputs $d_{k}$ expressed in equation (4).

$$
d_{k}=f\left(W^{(l a b e l)} y+b^{(l a b e l)}\right)
$$

The correct signal is one hot vector.

$$
t=[0, \ldots, 0,1,0, \ldots, 0]^{t}
$$

The error function is cross-entropy error $E_{c e}$ expressed in (6).

$$
E_{c e}(y, t)=-\sum_{k=1}^{K} t_{k} \log d_{k}(y)
$$

Figure 1 lists the model parameters and error functions of RAE. While AE aims to obtain a condensed vector representation best reproducing two child nodes of neighboring words or phrases, the whole RAE aims to classify the utterance intent accurately. Accordingly, the total error function is set as a weighted sum of two error functions in equation (7).

$$
E=\alpha E_{r e c}+(1-\alpha) E_{c e}
$$

The training of RAE optimizes the model parameters in accordance with a criterion of minimizing the total error function for all training data.

\subsection{Rule-based Syntactic Untying of RAE}

To relax the difference of the nonlinear operation depending on types of nodes, we designed a rule to switch two AEs depending on types of two child nodes manually. At the leaf level of a tree, most of words are nouns, while a sentence or a phrase is composed of a predicate with a subject or an object or a complement. The operation of vectors between words and noun phrases, and that between phrases and clauses are assumed to differ considerably. Hence, the manual rule switches two AEs, one for words and noun phrases, and the other for phrases and clauses. Along a tree, the

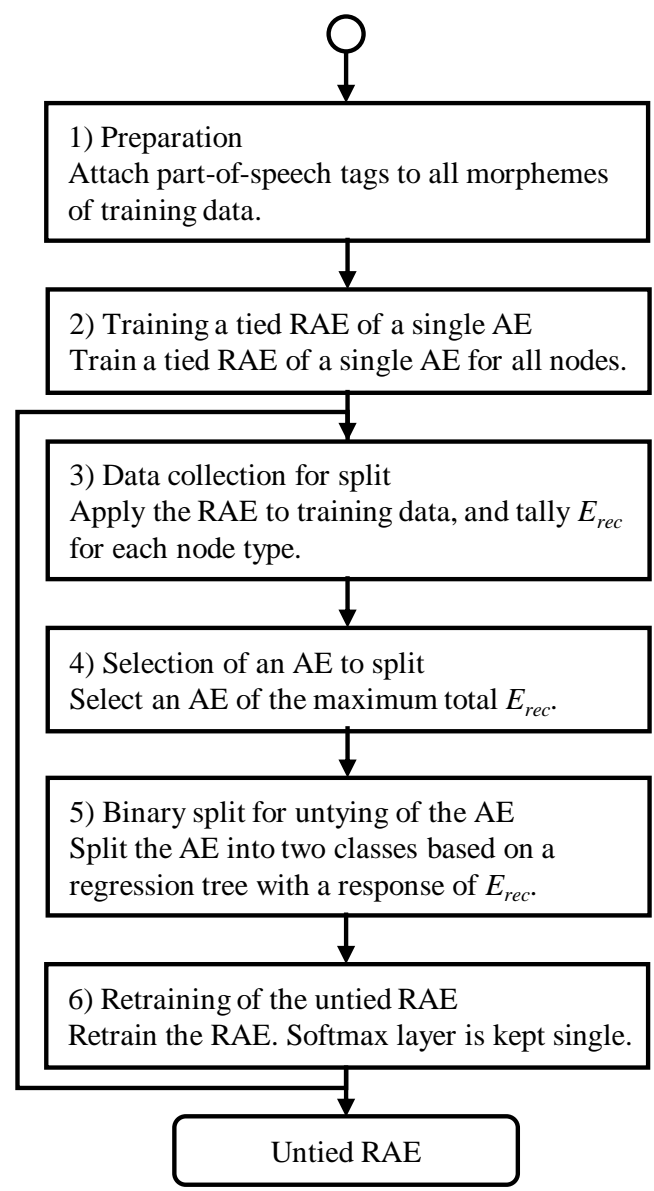

Figure 2: Procedure for training RAE of multiple AEs with data-driven untying

AE for words and noun phrases is applied at lower nodes around leaves, and the AE for phrases and clauses is applied at upper nodes close to the root node.

The node type is determined as follows. At leaf nodes, every word of a sentence is given a part-ofspeech tag as a node type by Japanese morpheme analyzer (Kudo et al., 2004). The number of tags is set at 10. At upper nodes, the node type is determined by the combination of node types of two child nodes. A look-up table of the node type is defined on the basis of Japanese grammar. Another look-up table determining which AE to apply on the basis of the node type is defined as well.

\subsection{Data-driven Untying of RAE}

To obtain a more effective untied RAE, we designed a training method including data-driven untying of RAE. The method is based on sequentially splitting an AE with regression trees to reduce the total reconstruction error $E_{\text {rec }}$. Specifically, the method splits an AE into two on the basis of a re- 
Table 2: Precision, recall, and accuracy of utterance intent classification of 65 classes

\begin{tabular}{l|ccc|ccc}
\hline method & \multicolumn{3}{|c|}{ training set } & \multicolumn{3}{c}{ test set } \\
\hline & prec. & recall & acc. & prec. & recall & acc. \\
(1) Cosine similarity of bag-of-words (BoW) & - & - & - & $76.0 \%$ & $74.2 \%$ & $\mathbf{8 5 . 1} \%$ \\
(2) Tied RAE based on random word vectors & $37.2 \%$ & $33.2 \%$ & $\mathbf{7 0 . 6 \%}$ & $32.0 \%$ & $65.6 \%$ & $\mathbf{6 6 . 4 \%}$ \\
(3) Tied RAE based on word2vec vectors & $81.2 \%$ & $78.8 \%$ & $\mathbf{8 8 . 7 \%}$ & $74.7 \%$ & $70.5 \%$ & $\mathbf{8 2 . 7 \%}$ \\
(4) RAE of two AEs untied by manual rule & $65.9 \%$ & $68.3 \%$ & $\mathbf{8 8 . 1 \%}$ & $63.0 \%$ & $62.5 \%$ & $\mathbf{8 4 . 0 \%}$ \\
(5) RAE of two AEs untied by data-driven split & $80.3 \%$ & $79.8 \%$ & $\mathbf{9 1 . 3 \%}$ & $72.4 \%$ & $72.3 \%$ & $\mathbf{8 5 . 6 \%}$ \\
(6) RAE of three AEs untied by data-driven split & $73.9 \%$ & $75.2 \%$ & $\mathbf{9 0 . 3 \%}$ & $70.8 \%$ & $67.9 \%$ & $\mathbf{8 4 . 8 \%}$ \\
\hline
\end{tabular}

gression tree with the response of the reconstruction error $E_{r e c}$, and optimizes the model parameters of split AEs alternatively.

Figure 2 shows the procedure. The procedure starts with giving a part-of-speech tag to every word of a sentence. While forming a tree, a unique node type is given according to the node types of child nodes. To be precise, a new node type is given to an unseen combination of node types of two child nodes, whereas the same node type is given when the combination of node types has been seen before.

Initially, a single tied AE for all node types is trained. Applying the $\mathrm{AE}$ to all training data, reconstruction error $E_{r e c}$ is tallied for each node type. Then, a class of all node types is split into two classes based on a regression tree of CART (Breiman et al., 1984) with the response of $E_{\text {rec }}$. The predictor variables are the node types of the left and right child nodes. Then, the AEs are retrained with L2 regularization after every binary split. Note that the softmax layer is kept single in order not to make the generated vector space completely different.

\section{Experiments}

\subsection{Experimental Setup}

An experiment of utterance intent classification was conducted with the annotated data described in Section 2. The number of classes was reduced to 65 by merging classes with few pieces of data with a similar class or into the others class. Considering the balance of frequent utterances and less-frequent ones, the frequencies of utterances were smoothed by applying a square root function. The numbers of utterances in the training and test sets were 7,833 and 870 , respectively. The ratio of unknown utterances in the test set was 15 percent.

\subsection{Conditions of Experiments}

Two types of word vectors, ramdom word vectors and word2vec vectors, were compared as the minimal elements of a tree. A total of 1.08 million word2vec vectors were trained with Japanese wikipedia texts of 1.1 billion words. The dimension of the vectors was fixed at 100 . The word $2 \mathrm{vec}$ vectors were trained by using skip-gram mode on the basis of results of preliminary experiments.

Three types of RAE, that is, a single tied AE, two AEs untied by the manual rule, and multiple AEs untied by the data-driven split, and a baseline method of cosine similarity of bag-of-words were evaluated.

\subsection{Experimental Results}

Table 2 shows the precision, recall, and accuracy of the classification for the training and test sets. The baseline method (1) showed relatively high performance, because the test set randomly chosen in consideration of the smoothed frequencies contained many known utterances and words seen in the training set. The tied RAE based on word2vec vectors (3) showed significantly better performance than the tied RAE based on random word vectors (2). While the RAE of two AEs untied by a manual rule (4) made a slight improvement, the RAE of two AEs untied by data-driven split (5) made more improvement. The resulting split was not simple, but one of the two AEs was to add a modifier, roughly speaking. However, the RAE of three AEs untied by data-driven split (6) showed a fall. We believe that the RAE was probably overlearned with thousands pieces of training data.

\section{Conclusions}

RAE was applied to utterance intent classification of a smartphone-based Japanese-language spoken dialogue system. To improve the classification accuracy, we examined the RAE of multiple AEs un- 
tied by a manual rule and RAEs of multiple AEs untied by data-driven split.

Comparing the untied RAEs of two AEs between the manual rule and data-driven split, the AEs untied by the data-driven split showed better accuracy. This means that splitting AEs based on a regression tree with the response of the reconstuction error is effective to some extent.

Reducing the model parameters effectively to circumvent overlearning, and utterance intent classification with more variations of utterances are future work.

\section{References}

L. Breiman, J. H. Friedman, R. A. Olshen, and C. J. Stone. 1984. Classification And Regression Trees. Chapman \& Hall CRC.

E. Guevara. 2010. A regression model of adjectivenoun compositionality in distributional semantics. Proc. of the 2010 Workshop on Geometrical Models of Natural Language Semantics 2010 pages 33-37.

D. Guo, G. Tur, W. Yih, and G. Zweig. 2014. Joint semantic utterance classification and slot filling with recursive neural networks. Proc. Spoken Language Technology Workshop 2014 pages 266-267.

T. Kudo, K. Yamamoto, and Y. Matsumoto. 2004. Applying conditional random fields to japanese morphological analysis. Proc. EMNLP 2004 pages 230 237.

T. Mikolov, I. Sutskever, K. Chen, G. Corrado, and J. Dean. 2013. Efficient estimation of word representation in vector space. arXiv: 1301.3781.

J. Mitchell and M. Lapata. 2010. Composition in distributional models of semantics. Cognitive Science 34(8):1388-1429.

J. Pennington, R. Socher, and C. D. Manning. 2014. Glove: Global vectors for word representation. Proc. EMNLP 2014 pages 1532-1543.

S. Ravuri and A. Stolcke. 2015. Recurrent neural network and lstm models for lexical utterance cllasification. Proc. Interspeech 2015 pages 135-139.

R. Socher, J. Bauer, C. D. Manning, and A. Y. Ng. 2013a. Parsing with compositional vector grammars. Proc. ACL 2013 pages 455-465.

R. Socher, J. Pennington, E. H. Huang, A. Y. Ng, and C. D. Manning. 2011. Semi-supervised recursive autoencoders for predicting sentiment distributions. Proc. EMNLP 2011 pages 151-161.

R. Socher, A. Perelygin, J. Y. Wu, J. Chuang, C. D. Manning, A. Y. Ng, and C. Potts. 2013b. Recursive deep models for semantic compositionality over a sentiment treebank. Proc. EMNLP 2013 pages 1631-1642.

R. Socher et al. 2012. Semantic compositionality through recursive matrix-vector spaces. Proc. EMNLP 2012 pages 1201-1211. 